\title{
Location of the positive charges in cationic amphiphiles modulates their mechanism of action against model membranes
}

Marcio M. Almeida, Katia R. Perez, Allison Faig, Kathryn E. Uhrich, Karin A. Riske
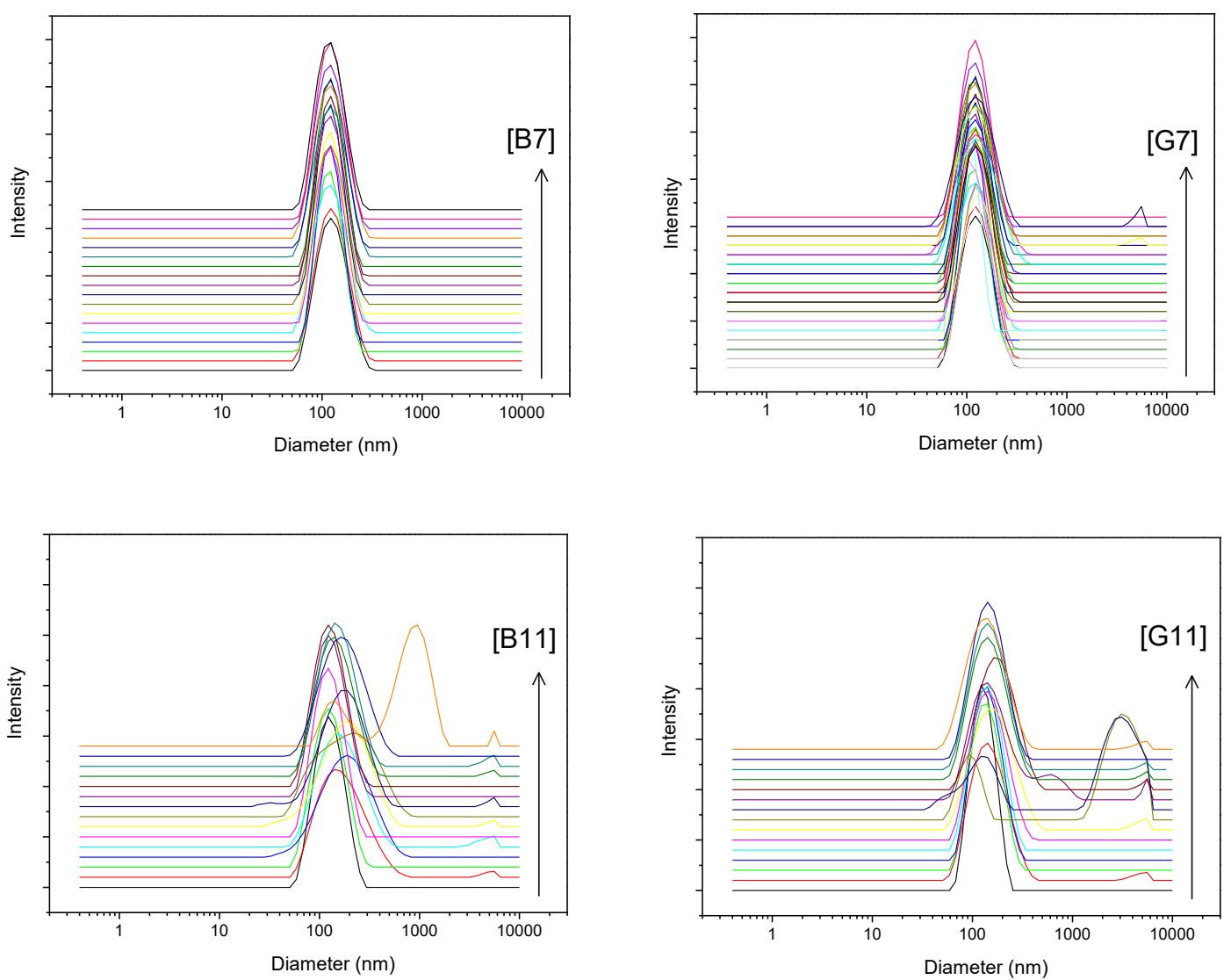

Figure S1 - Size distribution (intensity) measured along injections of each CAm into $0.1 \mathrm{mM}$ POPC:POPG 7:3 (LUVs). The curves are shifted for clarity and each one corresponds to the Zaverage sizes shown in Figure 1. The arrows indicate the direction of increase in CAm concentration. Measurements were performed at $25^{\circ} \mathrm{C}$. 

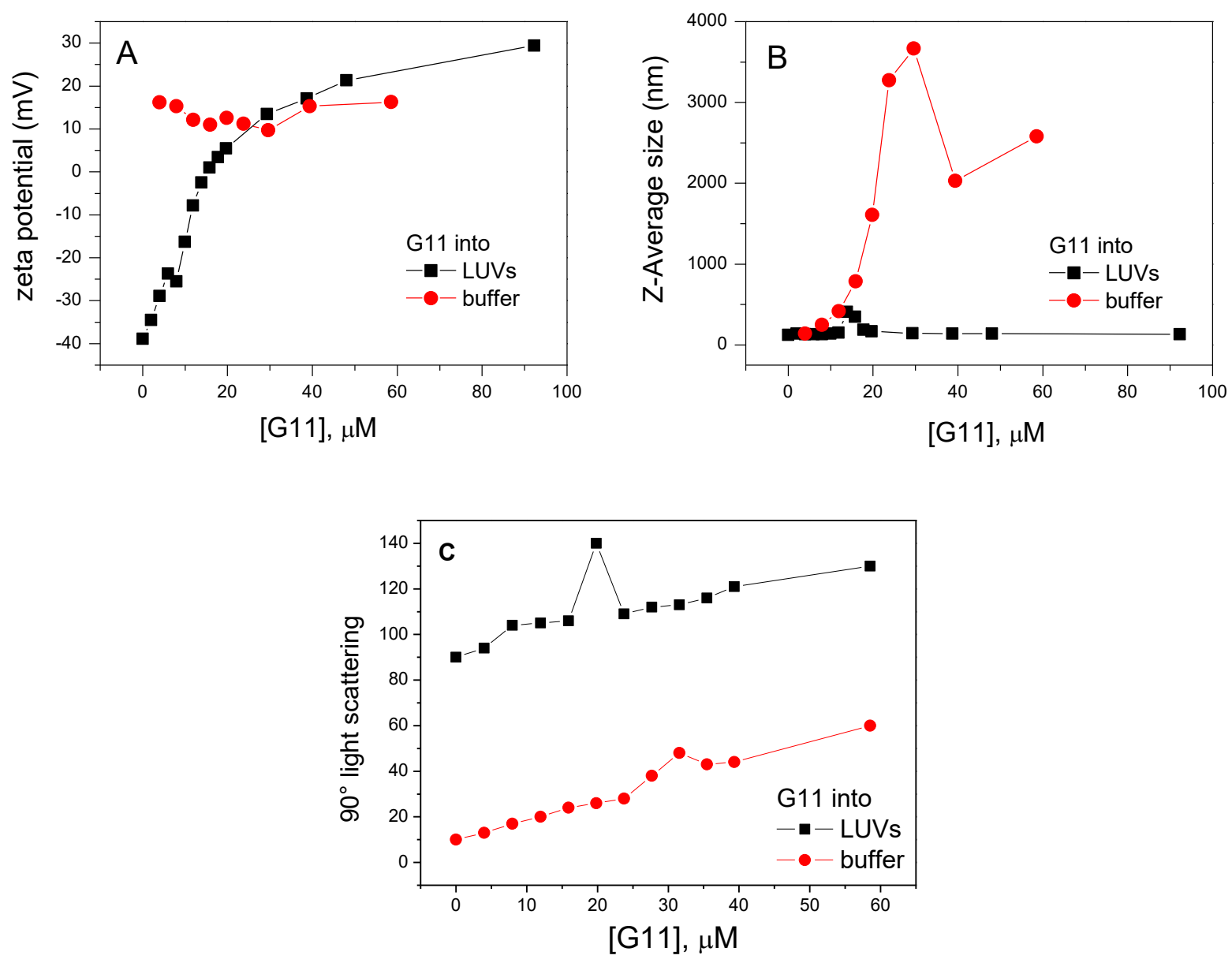

Figure S2 - Zeta potential (A), Z-Average size (B) and $90^{\circ}$ light scattering (C) measured along injections of G11 into buffer and 0.1 mM POPC:POPG 7:3 (LUVs). 90 static light scattering was measured in an Agilent Cary Eclipse spectrofluorimeter (Santa Clara, CA) at $\lambda=500 \mathrm{~nm}$. The sample (buffer or 0.1 mM POPC:POPG 7:3 LUVs) was accommodated in a quartz cuvette and injections of a CAm stock solution (2.4 mM G11) were made and the light scattering intensity recorded for $1 \mathrm{~min}$. Measurements were performed at $25^{\circ} \mathrm{C}$. 


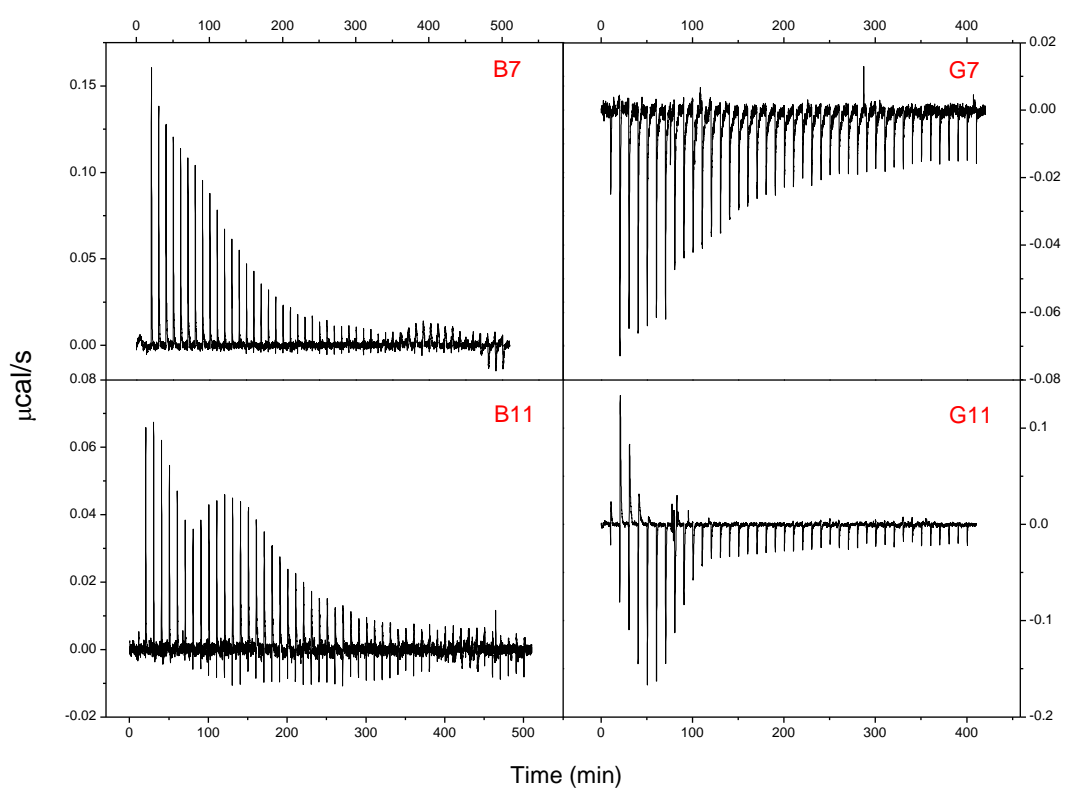

Figure S3 - Heat flows obtained from ITC experiments. The sample cell contains $20 \mu \mathrm{M}$ CAm and the syringe was loaded with POPC:POPG 7:3 LUVs (5 mM lipid concentration). Consecutive $5 \mu \mathrm{L}$ injection were done every $10 \mathrm{~min}$. The temperature was $25^{\circ} \mathrm{C}$.

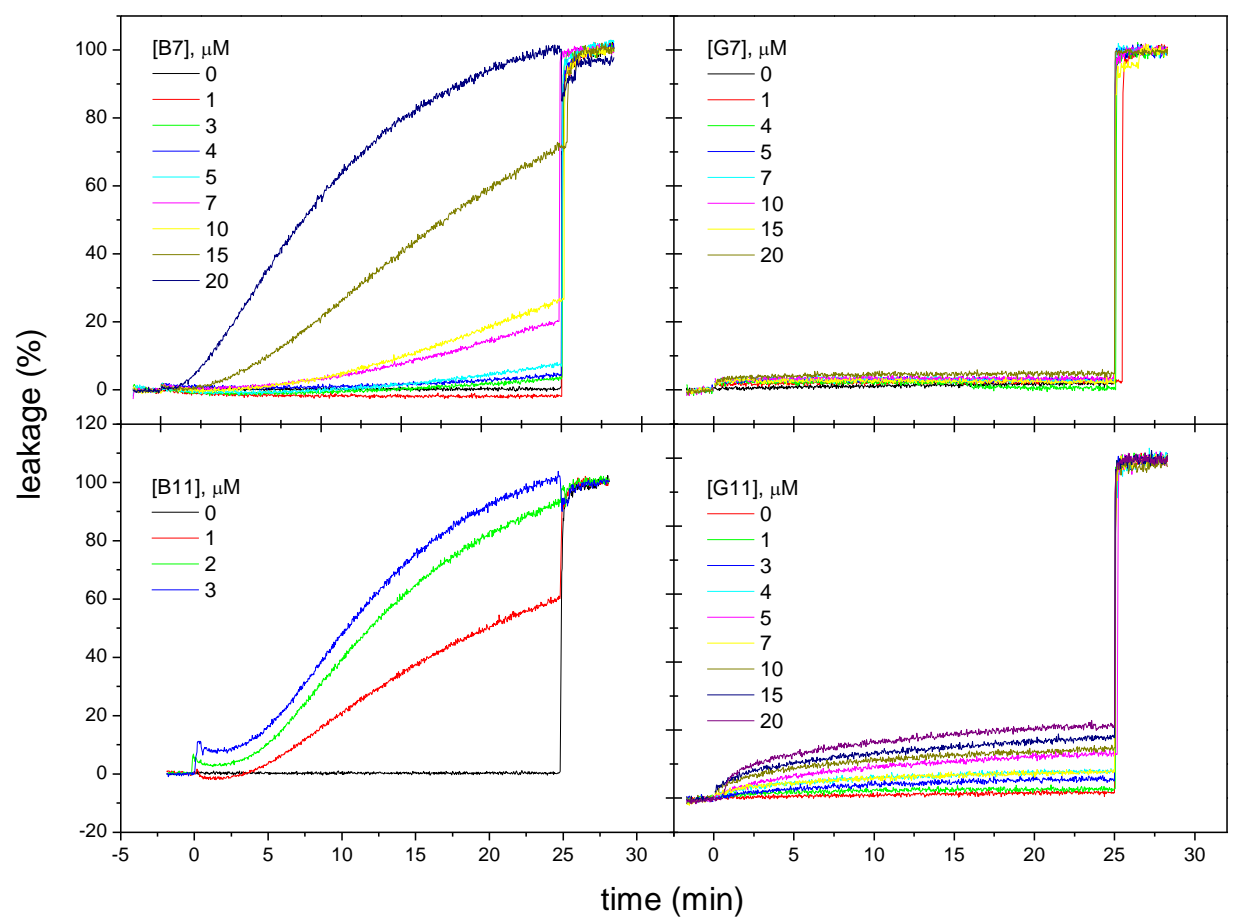

Figure S4 - Kinetics of CF fluorescence intensity curves (normalized as explained in the materials and methods section) obtained from POPC:POPG 7:3 (22 $\mu \mathrm{M}$ lipid concentration) encapsulating 50 $\mathrm{mM} \mathrm{CF}$. The CAms (concentrations indicated in the figure legend) were injected at 0 min and Triton X-100 was added 25 min after to induce full leakage. 

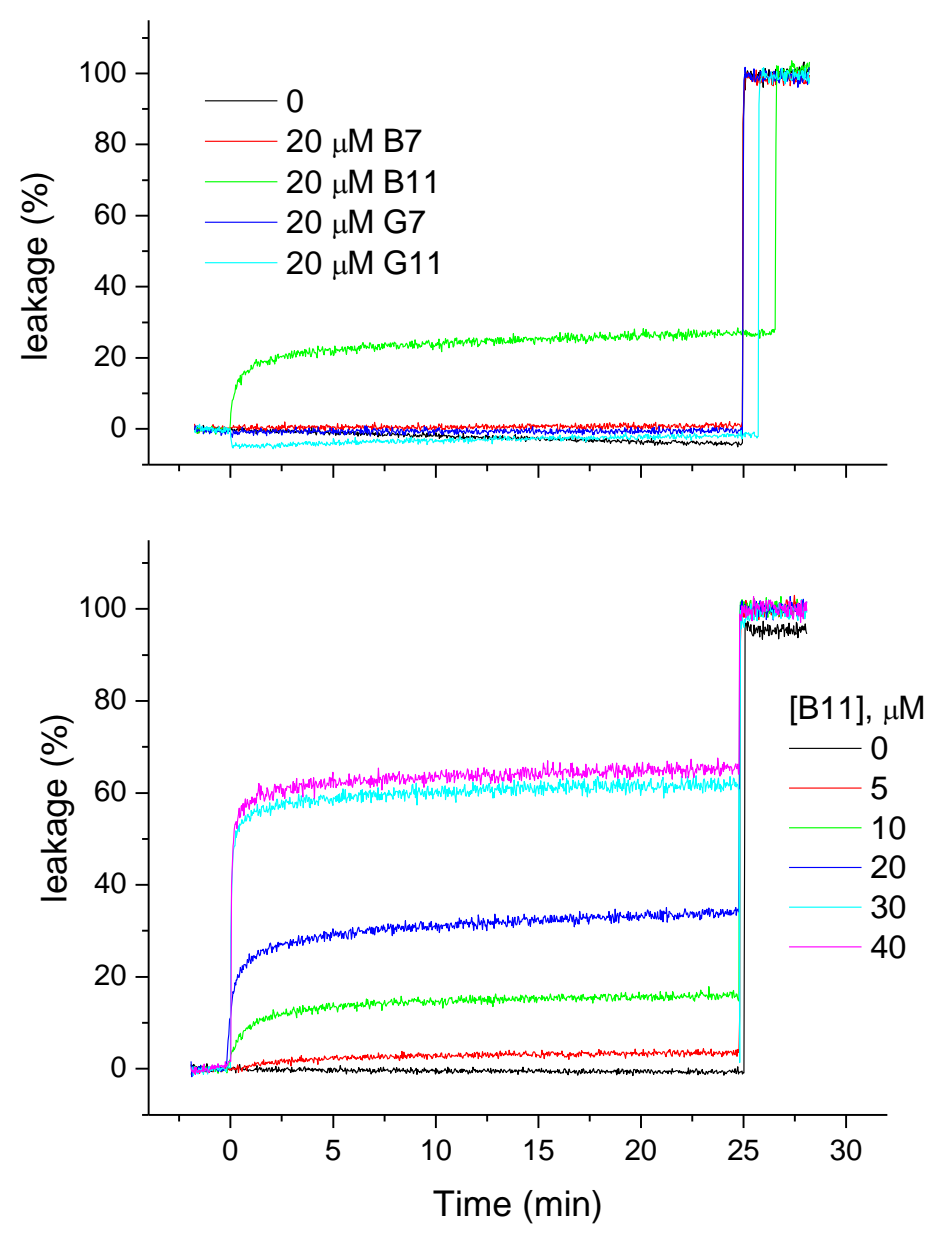

Figure S5 - Kinetics of CF fluorescence intensity curves (normalized as explained in the materials and methods section) obtained from POPC (40 $\mu \mathrm{M}$-top- or 32-bottom- $\mu \mathrm{M}$ lipid concentration) encapsulating $50 \mathrm{mM} \mathrm{CF}$. The CAms (concentrations indicated in the figure legend) were injected at $0 \mathrm{~min}$ and Triton $\mathrm{X}-100$ was added $25 \mathrm{~min}$ after to induce full leakage. 